\title{
Design of organic molecules and molecular assemblies with electron spin alignment
}

\author{
Hiizu Iwamura \\ Department of Applied Nolecular Science, Institute for Molecular Science, \\ Myodaiji, Okazaki 444, Japan
}

\begin{abstract}
The electron spin-spin interaction between the two triplet diphenylcarbene units incorporated in the [2.2] paracyclophane skeleton has been investigated by ESR spectroscopy. The pseudoortho and pseudopara bis (phenylmethyleny1) [2.2] paracyclophanes are in the ground quintet state, while the pseudometa isomer is singlet. A conformational analysis of these high-spin dicarbenes has been carried out by examining their dipolar interaction tensors. The McConnell's theory on the ferromagnetic interaction between two organic radicals has been demonstrated and a new strategy for increasing the dimension of high-spin aromatic molecules was found. On the basis of these findings, the molecular aggregates of some p-alkoxy substituted diphenylcarbenes were generated in the crystals of the precursor diazodiphenylmethanes to give 4 to 8 spins aligned among the neighboring triplet molecules.
\end{abstract}

\section{INTRODUCTION}

The large majority of organic molecules have closed shell electronic structure, i.e., they have singlet ground state with equal number of electrons having $\alpha$ and $\beta$ spin. Most organic compounds are therefore diamagnetic. There are some organic molecules that have open-shell structures where not all electrons are paired. Many of these molecules have one odd electron and therefore doublet ground state. These free radicals are paramagnetic. The alignment of spins in molecular systems becomes an issue when there is an interaction between two doublet centers as in diradicals and radical pairs. The Coulombic repulsion between electrons lifts the zeroth-order degeneracy and gives rise to singlet and triplet states of different total energy for these chemical entities. It is highly important in the chemistry of diradicals (ref. 1) and radical pairs (ref. 2) which state lies lower in energy. The conditions for having the ground triplet states and the importance of the singlet-triplet radical pairs in free radical reactions have been amply discussed.

While diradicals and radical pairs are accepted as important reaction intermediates, the chemistry of these and more highly spin-aligned systems is considered also to be the best area where one can look for interesting magnetic properties from organic materials. Recently we have shown that polycarbenes 1 and 2 generated by photolysis of the corresponding polydiazo compounds have their highest possible spin multiplicities in the ground states (ref. 3). The magnetic susceptibility measurement has revealed that nonet tetracarbene $1(n=4)$ has a paramagnetic property of very large magnetic moments $(S=8 / 2)$ (ref. 4). Higher homologs of $\underset{\sim}{1}$ and 2 are expected to be best characterized as organic molecular superparamagnets (ref. 5). Curie plots of the paramagnetic susceptibility of $\underset{\tilde{n}}{1}(\mathrm{n}=4)$ in 2-methyltetrahydrofuran glass or in polycrystalline sample have shown, however, tha th the intermolecular interaction in the fortuitously formed aggregates under these conditions is antiferromagnetic. It appears to be the rule rather than exception that the intermolecular interaction between open-shell organic

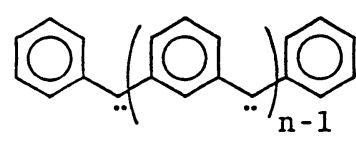

$\underset{\sim}{1}(n=3,4,5,6)$

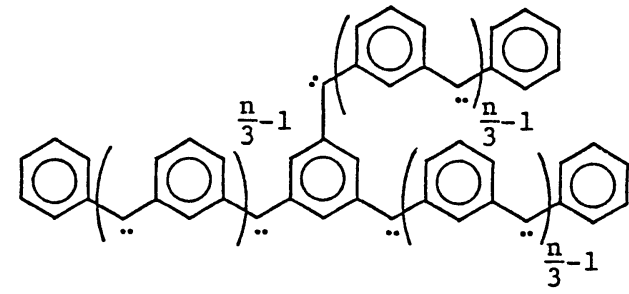

$\underset{\sim}{2}(n=3,6)$ 
molecules should be antiferromagnetic. Therefore, it is imperative for obtaining the spin alignment in macroscopic scale throughout the molecular assembly to design systems in which molecular interaction could become ferromagnetic. There appear to be a couple of plausible methods for achieving this objective. One is to take advantage of a weak hyperconjugative interaction through a short chain connecting high-spin molecules (ref. 6). The other is a design of molecular stacking of spin-distributed aromatic rings leading to intermolecular spin alignment (ref. 7). In this paper, we would like to discuss the latter strategy on the basis of the NicConnell's theory (ref. 8) and show a partial success of the application of this method.

\section{DESIGN OF A MODEL FOR FERROMAGNETIC MOLECULAR STACKING OF DIPHENYLCARBENE UNITS}

Aromatic molecules have a tendency to stack in organized systems. It would therefore be desirable if requirements for parallel spins, i.e., ferromagnetic stacking between two aromatic radicals are found. For a set of carbenes A and B each initially in a triplet state, the phenomenological interaction Hamiltonian is written out as

$$
\hat{\mathrm{H}}^{\mathrm{AB}}=-2 \sum_{\mathrm{m}, \mathrm{n}=1,2} \mathrm{~J}_{\mathrm{mn}}^{\mathrm{AB}} \mathbf{s}_{\mathrm{m}}^{\mathrm{A}} \cdot \mathbf{s}_{\mathrm{n}}^{\mathrm{B}}
$$

where $J_{\mathrm{mn}}^{\mathrm{AB}}$ is the valence-bond exchange integral between the odd electrons $\mathrm{m}$ and $\mathrm{n}$ on carbenes $A$ and $B$, respectively, with respective spin angular momentum operators $\mathbf{S}_{\mathrm{m}}^{\mathrm{A}}$ and $\mathbf{S}_{\mathrm{n}}^{\mathrm{B}}$. For weak $\pi$-type interactions, we express the operator $\mathbf{S}^{A} \cdot \mathbf{S}^{B}$ as

$$
-2 J^{A B} \mathbf{s}^{A} \cdot \mathbf{s}^{B}=-J^{A B}\left(\mathbf{s}^{2}-\mathbf{s}^{A^{2}}-\mathbf{s}^{B 2}\right)
$$

where $\mathbf{S}=\mathbf{S}^{\mathrm{A}}+\mathbf{S}^{\mathrm{B}}$ is the operator for the total spin angular momentum, with eigenvalue $S$. For two spins $\mathbf{S}^{\mathrm{A}}$ and $\mathbf{S}^{\mathrm{B}}$ with eigenvalues $S^{\mathrm{A}}=1$ and $S^{\mathrm{B}}=1$, the expectation value of $\hat{\mathrm{H}}$ is

$$
E=-J[S(S+1)-4]
$$

The state of eigenvalue $-2 J$ has five-fold degeneracy with a total $\operatorname{spin} S=2$, which is a quintet state. The eigenfunctions of the quintet state are given by eq. 4 , where the subscript $i$ of $Q_{i}$ shows the eigenvalue of $s_{z}$.

$$
\begin{aligned}
& \ell_{2}=\alpha \alpha \alpha \alpha \\
& \ell_{1}=(1 / 2)[\alpha \alpha(\alpha \beta+\beta \alpha)+(\alpha \beta+\beta \alpha) \alpha \alpha] \\
& \ell_{0}=(1 / \sqrt{6})[\alpha \alpha \beta \beta+(\alpha \beta+\beta \alpha)(\alpha \beta+\beta \alpha)+\beta \beta \alpha \alpha] \\
& \ell_{-1}=(1 / 2)[(\alpha \beta+\beta \alpha) \beta \beta+\beta \beta(\alpha \beta+\beta \alpha)] \\
& \ell_{-2}=\beta \beta \beta \beta
\end{aligned}
$$

The state of eigenvalue $2 J$ is three-fold degenerate (eq. 5) with a total spin $S=1$.

$$
\begin{aligned}
& T_{1}=(1 / 2)[\alpha \alpha(\alpha \beta+\beta \alpha)-(\alpha \beta+\beta \alpha) \alpha \alpha] \\
& T_{0}=(1 / \sqrt{2})(-\alpha \alpha \beta \beta+\beta \beta \alpha \alpha) \\
& T_{-1}=(1 / 2)[(\alpha \beta+\beta \alpha) \beta \beta-\beta \beta(\alpha \beta+\beta \alpha)]
\end{aligned}
$$

The eigenfunction which belongs to the eigenvalue $4 \mathrm{~J}$ is a singlet state $(S=0)$ and given by eq. 6 .

$$
s_{0}=(1 / \sqrt{3})[\alpha \alpha \beta \beta-(1 / 2)(\alpha \beta+\beta \alpha)(\alpha \beta+\beta \alpha)+\beta \beta \alpha \alpha]
$$

Since the valence-bond exchange integral $J^{\mathrm{AB}}$ is usually negative between open-shell organic molecules at a distance of the van der Waals contact, the ground state tends to become singlet as in Fig. 1b. In order to develop macroscopic ferromagnetism, it is highly necessary to find the conditions under which case Fig. 1a could result; the intermolecular interaction between high-spin molecules has to be ferromagnetic. How can this be designed? There is one such prediction by McConnell (ref. 8). According to this theory, an effective exchange interaction between two free radicals can be ferromagnetic when the product of spin densities $\rho_{i}$ and $\rho_{j}$ at two interacting sites $i$ and $j$ on different molecules $A$ and $B$ is negative in sign, since the valence-bond exchange integral $J_{i j}$ is negative. (eq. 7).

$$
\hat{\mathrm{H}}^{\mathrm{AB}}=-2 \sum_{i j} J_{i j}^{\mathrm{AB}} \mathbf{s}_{i}^{\mathrm{A}} \cdot \mathbf{s}_{j}^{\mathrm{B}}=-2 \mathbf{s}^{\mathrm{A}} \cdot \mathbf{s}^{\mathrm{B}} \sum_{i j} J_{i j}^{\mathrm{AB}} \rho_{i}^{\mathrm{A}} \rho_{j}^{\mathrm{B}}
$$

Whereas the theory has been referred to interpreting complicated magnetic interactions found in crystals of stable organic radicals (ref.9), it has never been tested using a logical model system.

Let us take parallel stacking of the two benzene rings one from each triplet diphenylcarbene molecule. Since the $\pi$-spin distribution in the latter is as shown in Fig. 2 (ref. 10), the 


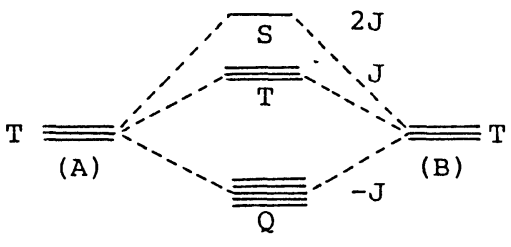

(a) $\mathrm{J}>0$

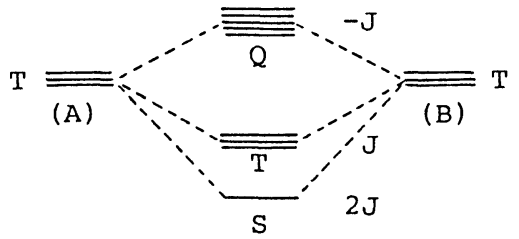

(b) $J<0$

Fig. 1. Interaction diagrams for two triplet species A and B (ref. 11)<smiles>C1CCC(C2CCCCO2)CC1</smiles>

$$
\begin{array}{cccc}
1 & +0.590 & 4 & -0.039 \\
& (+0.733) & 5 & +0.111 \\
2 & -0.022 & 6 & -0.038 \\
3 & +0.101 & 7 & +0.122
\end{array}
$$

Fig. $2 \pi$-Spin distribution in diphenylcarbene (ref. 10)

sign of the $\rho_{i} \rho_{j}$ at each interacting site between the two benzene rings is all negative in the ortho and para stacking patterns and all positive in the gem and meta (Fig. 3 ).

Therefore, the ortho and para overlaps are predicted to give quintet ground states, while the gem and meta would lead to the ground singlet state. As a model system to test this theory, we have taken advantage of the [2.2] paracyclophane skeleton. Among the three isomers of bis(phenylmethyleny1) [2.2] paracyclophanes $\underset{\sim}{3} \sim \underset{\sim}{5}$, the pseudoortho and pseudopara isomers satisfy the McConnell's condition to give the qüintet ground state.

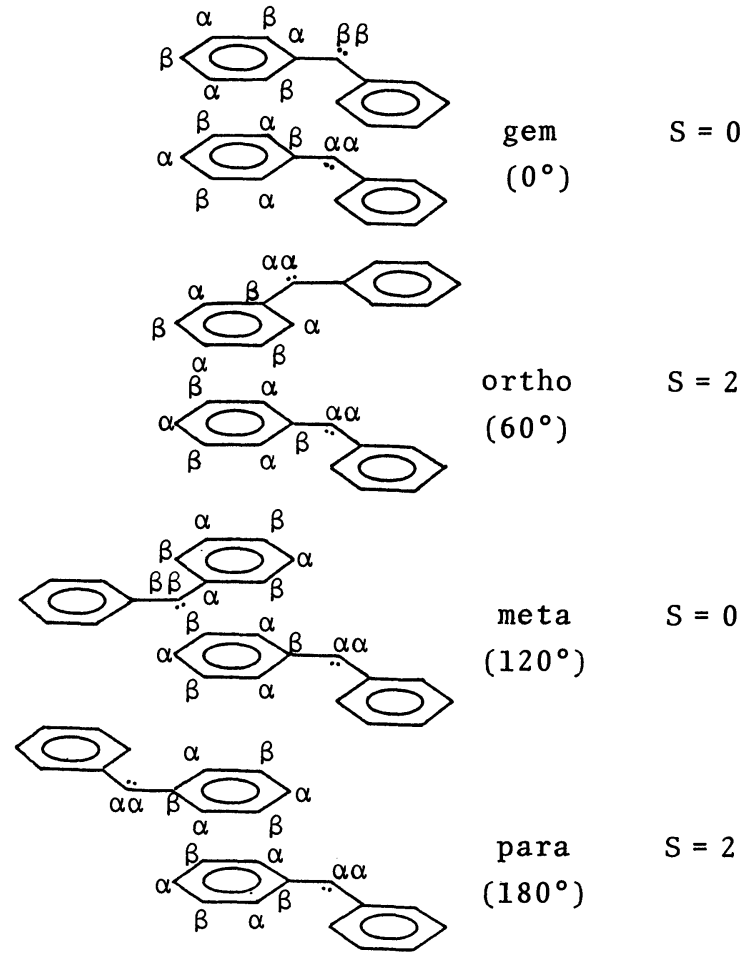

Fig. 3. Stacking patterns of the two benzene rings one from each diphenylcarbene molecule pseudoortho

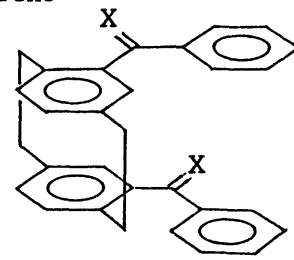

$\underset{\sim}{3}(\mathrm{X}=:), \underset{\sim \sim}{3 \mathrm{~b}}(\mathrm{X}=0), \underset{\sim \sim}{3 \mathrm{~d}}\left(\mathrm{X}=\mathrm{N}_{2}\right)$

pseudometa

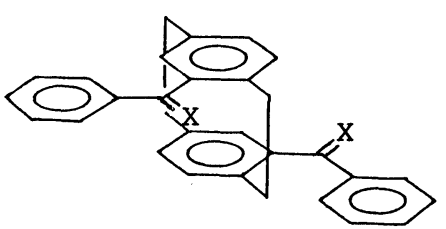

$\underset{\sim}{4}(\mathrm{X}=:), \underset{\sim \sim}{4 \mathrm{~b}}(\mathrm{X}=0), \underset{\sim \sim}{4 \mathrm{~d}}\left(\mathrm{X}=\mathrm{N}_{2}\right)$

pseudopara

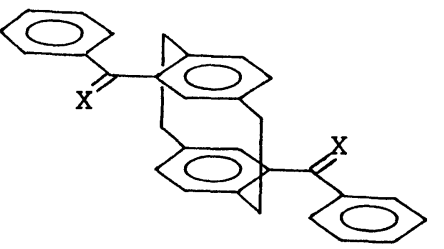

$\underset{\sim}{5}(\mathrm{X}=:), \underset{\sim \sim}{5 \mathrm{~b}}(\mathrm{X}=0), \underset{\sim \sim}{5 \mathrm{~d}}\left(\mathrm{X}=\mathrm{N}_{2}\right)$ 

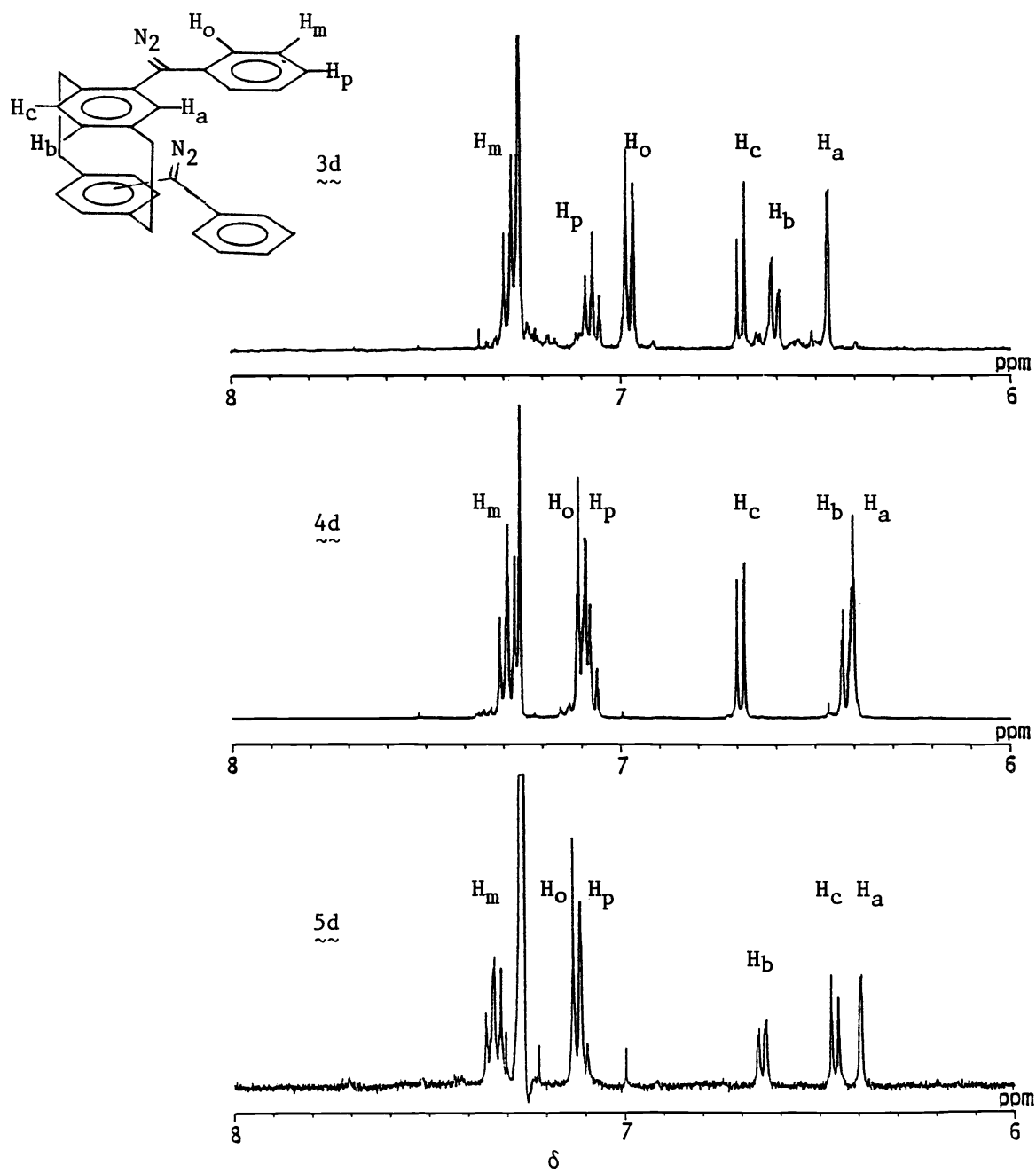

Fig. 4. Partial $400 \mathrm{MHz}{ }^{1} \mathrm{H}$ NMR spectra of didiazo compounds $\underset{\sim \sim}{3 \mathrm{~d}}, \underset{\sim \sim}{4 \mathrm{~d}}$ and $\underset{\sim \sim}{5 \mathrm{~d}}$

\section{PREPARATION OF ISOMERIC BIS $(\alpha-$ DIAZOBENZYL)[2.2]PARACYCLOPHANES}

Isomeric dibromo[2,2] paracyclophanes 3a $\sim$ 5 a were obtained by bromination of [2.2] paracyclophane (ref. 12). The dilithio compouñ̃ s obtained from $3 \mathrm{a} \sim{ }_{\sim}$ a a on treatment with n-buty1lithium in ether were allowed to react with benzaldehyde to give the corresponding diols. The psudoortho and pseudometa diols were oxidized with pyridinium chlorochromate in $\mathrm{CH}_{2} \mathrm{Cl}_{2}$ to give diketones $3 \mathrm{~b}$ and $4 \mathrm{~b}$. Pseudopara diol was smoothly oxidized with sodium dichromate in acetic acid. Kẽtones $\underset{\sim}{\tilde{b}} \tilde{\mathrm{b}} \sim \underset{\sim}{5} \mathrm{~b}$ were resistant to hydrazine hydrate under typical conditions. The corresponding dihydrazones $3 c \sim 5 c$ were obtained by the reaction with excess anhydrous hydrazine and hydrazine monohydroch1oride in DMSO at $110^{\circ} \mathrm{C}$ for $10 \mathrm{~h}$. Didiazo compounds ${\underset{\sim}{\sim}}_{\sim} \sim$ $5 \mathrm{~d}$ were obtained by oxidation of $3 \mathrm{c} \sim 5 \mathrm{c}$ with yellow $\mathrm{HgO}$ and purified by short-column chromatography on alumina: pseudoortho, mp 107-109 ${ }^{\circ} \mathrm{C} \mathrm{dec}$; pseudometa, mp $136-138{ }^{\circ} \mathrm{C} \mathrm{dec}$; pseudopara, mp $133-135{ }^{\circ} \mathrm{C}$ dec. Their $1_{\mathrm{H}}$ NMR spectra are shown in Fig. 4.

\section{ESR DETECTION OF ISOMERIC \\ BIS(PHENYLMETHYLENYL)[2.2] PARACYCLOPHANES AND DETERMINATION OF THEIR SPIN STATES}

\section{Pseudoortho-bis(phenylmethylenyl)[2.2]paracyclophane}

A solid solution of $3 \mathrm{~d}$ in 2-methyltetrahydrofuran (2-MTHF) was irradiated with Pyrex-filtered light in an ESR cavity at $11 \mathrm{~K}$. A set of intense ESR signals were obtained as shown in Fig. $5 \mathrm{a}$. The spectrum as a whole resembles that of m-phenylenebis (phenylmethylene) $\left(\frac{1}{\sim}(n=2)\right.$ ) in the quintet ground state. The resonance positions and intensities of the signals are 


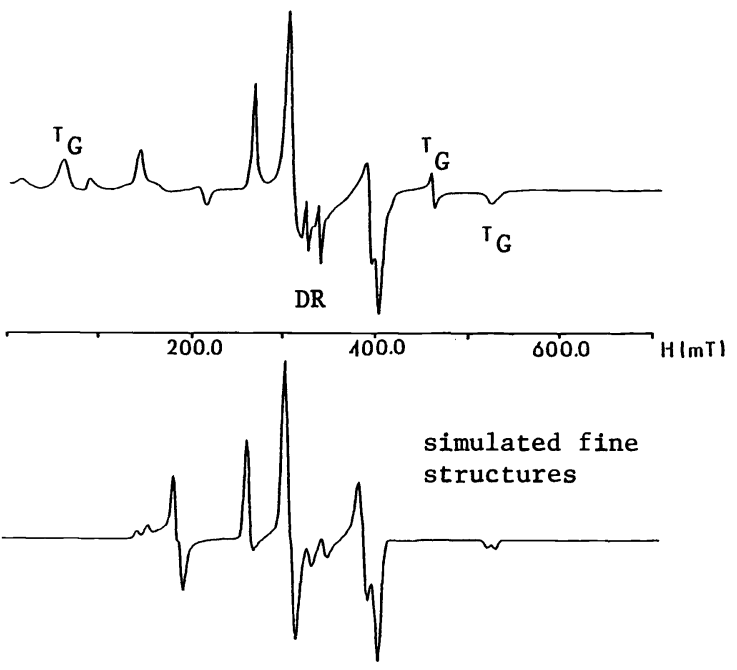

Fig. 5. ESR spectrum obtained on a Varian E-112/V7800 spectrometer with an $\mathrm{X}$-band microwave unit by irradiating pseudoortho didiazo compound $3 \mathrm{~d}$ in 2-MTHF at $11 \mathrm{~K}$. Signals $\mathrm{T}$ and $\mathrm{R}$ are assigned to a monocarbene and $\tilde{a}$ doublet radical, respectively, formed adventitiously. See text for the simulated spectrum.

reproduced reasonably well by a second-order perturbational calculation as a quintet species with zero-field splitting parameters $|D|=0.0624 \mathrm{~cm}^{-1}$ and $|\mathrm{E}|=0.0190 \mathrm{~cm}^{-1}$ (Fig. $5 \mathrm{~b}$ ). The fitting between the observed and calculated spectra is not satisfactory for transitions at the lower magnetic field than $200 \mathrm{mT}$. This is due to the limitation of the high-field approximation used in the calculation (ref. 13). In addition to these quintet signals, triplet signals $\left(\mathrm{T}_{\mathrm{G}}\right)$ were detected at $62.8,463.0,526.7$ and $732.0 \mathrm{mT}$ and assigned to a monocarbene derived from $3_{\sim}^{\mathrm{d}}\left(|\mathrm{D}|=0.3730 \mathrm{~cm}^{-1}\right.$ and $\left.|\mathrm{E}|=0.0156 \mathrm{~cm}^{-1}\right)$. Another triplet species

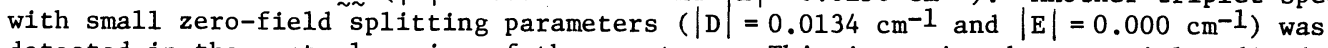
detected in the central region of the spectrum. This is assigned to a triplet diradical (DR) formed presumably from dicarbene 3 .

In order to determine the ground state spin multiplicity and thermal stability of the observed species, the temperature dependence of the signal intensities was studied (Fig. 6). The signal intensities of the quintet (Q) were found to obey Curie law in the temperature range $11 \sim 50 \mathrm{~K}$, indicating that the pseudoortho dicarbene 3 has the quintet ground state. This is the first experimental demonstration that the magnẽtic interaction between two diphenylcarbene moieties becomes ferromagnetic when two spin-containing benzene rings one from each diphenylcarbene are stacked in a proper mode dictated by McConnell (ref. 8). The signals due to $\mathrm{T}_{\mathrm{G}}$ and $\mathrm{DR}$ also obeyed the Curie law in the temperature ranges $11 \sim 85 \mathrm{~K}$ and $11 \sim$ $50 \mathrm{~K}$, respectively. At above $50 \mathrm{~K}$, the latter signal intensities increased irreversibly at the expense of $Q$ species, suggesting generation of $D R$ from $Q$.

At temperatures higher than $20 \mathrm{~K}$, a new signal started to appear at $104.0 \mathrm{mT}$. The intensity of the signal increased as the temperature was elevated and reached a maximum at $55 \mathrm{~K}$ (Fig.6). The signal decayed irreversibly at temperatures higher than $50 \mathrm{~K}$. The thermal behavior is characteristic of a thermally populated species $\left(\mathrm{T}_{\mathrm{T}}\right)$. Since relation $\mathbf{D}_{\mathrm{T}}=-3 \mathbf{D}_{\mathrm{Q}}$ holds for two equivalent triplet species interacting weakly (ref. 11), the zero-field splitting parameters of the thermally populated triplet are estimated to be $|D|=0.1872 \mathrm{~cm}^{-1}$ and ||$E \mid=0.0570 \mathrm{~cm}^{-1}$. The transition field of $\mathrm{H}_{\mathrm{min}}$ for $\mathrm{T}_{\mathrm{T}}$ is calculated to be $104.9 \mathrm{mT}$ from the estimated $\mathrm{D}$ and $\mathrm{E}$ values in good agreement with the observed value of $104.0 \mathrm{mT}$. Since the signals due to the other transitions of $\mathrm{T}_{\mathrm{T}}$ were weak and overlapped with intense $\mathrm{Q}$ signals, they could not be identified precisely. In reference to Fig. la and by assuming a Boltzman distribution of electron spins among the three states, the temperature dependence of the signal intensity for $\mathrm{T}_{\mathrm{T}}$ is given by eq. 8 .

$$
I\left(\mathrm{~T}_{\mathrm{T}}\right)=\frac{\mathrm{C} \exp \left(-\Delta E_{\mathrm{QT}} / R T\right)}{5+3 \exp \left(-\Delta E_{\mathrm{QT}} / R T\right)+\exp \left(-3 \Delta E_{\mathrm{QT}} / 2 R T\right)} \cdot \frac{1}{T}
$$

where $\Delta E_{\mathrm{OT}}$ is the energy gap between the ground $Q$ and the thermally populated $T$ states. The data in Fig. 6 give $\Delta E_{\mathrm{QT}}=61 \mathrm{~cm}^{-1}(=175 \mathrm{cal} / \mathrm{mol})$ and $J$ is calculated from eq. 2 to be +16 $\mathrm{cm}^{-1}$. 


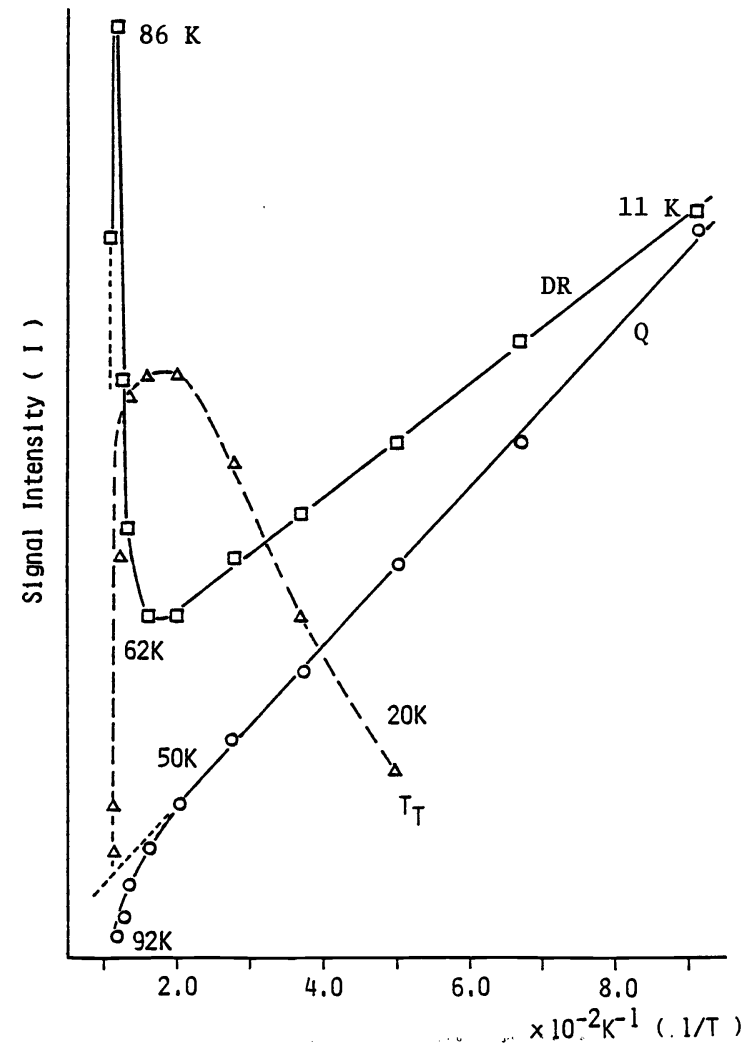

Fig. 6. Plots of ESR signal intensities vs the reciprocals of temperature for pseudoor tho dicarbene 3 .

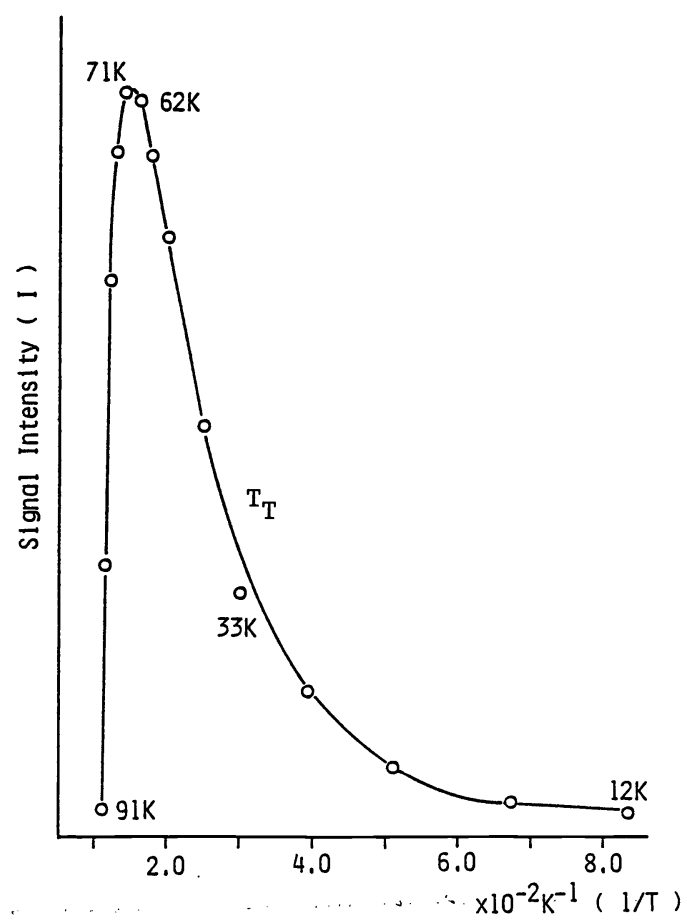

Fig. 8. Plots of ESR signal intensities vs the reciprocals of temperature for pseudometa dicarbene $\underset{\sim}{4}$.

\section{Pseudometa-bis(phenylmethylenyl)[2.2]paracyclophane}

In sharp contrast with the pseudoortho isomer, signals due to the quintet species were not detected in the ESR spectrum obtained after irradiation of didiazo compound $4 \mathrm{~d}$ at $11 \mathrm{~K}$. Instead a set of signals due to a monocarbene $\left(|D|=0.3793 \mathrm{~cm}^{-1}\right.$ and $|\mathrm{E}|=0.0158 \mathrm{~cm}^{-1}$ ) were found. When the temperature of the irradiated solid solution was raised to $25 \mathrm{~K}$, a new signal started to appear at $119.4 \mathrm{mT}$ and its intensity increased with temperature giving a maximum at $65 \mathrm{~K}$. Two additional peaks at 209.1 and $425.4 \mathrm{mT}$ were discernible at this temperature (Fig. 7). The resonance fields of the three signals correspond to a triplet species $\left(\mathrm{T}_{\mathrm{T}}\right.$ ) with $|\mathrm{D}|=0.184 \mathrm{~cm}^{-1}$ and $|\mathrm{E}|=0.0023 \mathrm{~cm}^{-1}$. Their thermal behavior (Fig. 8) is consistent with a thermally populated species.

a

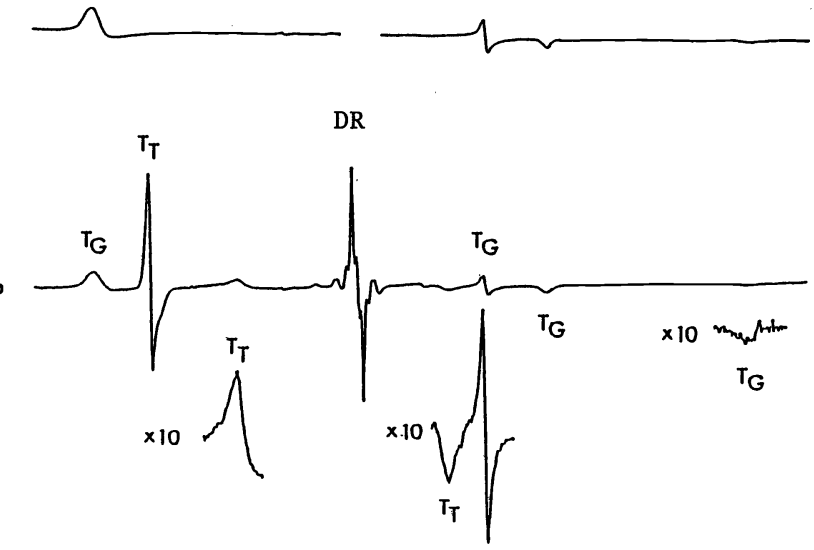

$200.0-400.0-600.0 \mathrm{HImTI}$

Fig. 7. ESR spectra obtained (a) in the dark after generation of pseudometa dicarbene 4 at $11 \mathrm{~K}$, and (b) when sample (a) was warmed to $60 \mathrm{~K}$. DR is due to a diradícal formed adventitiously 
Since quintet signals were not detected and the observed triplet is thermally populated, the two diphenylcarbene units in 4 are concluded to interact antiferromagnetically to give a singlet ground state. The energy difference $\left(\Delta E_{S T}\right)$ between the ground singlet and higherlying triplet states (see Fig. $1 \mathrm{~b}$ ) is given by eq. 9:

$$
I\left(\mathrm{~T}_{\mathrm{T}}\right)=\frac{\mathrm{C} \exp \left(-\Delta E_{\mathrm{ST}} / R T\right)}{1+3 \exp \left(-\Delta E_{\mathrm{ST}} / R T\right)+5 \exp \left(-3 \Delta E_{\mathrm{ST}} / R T\right)} \cdot \frac{1}{T}
$$

From the thermal behavior of the signal intensities of $\mathrm{T}_{\mathrm{T}}$ in the temperature range $11 \sim 65 \mathrm{~K}$, the $\Delta E_{\text {ST }}$ value is estimated to be $98 \mathrm{~cm}^{-1}(=280 \mathrm{cal} / \mathrm{mol})$, the $J$ value being $-49 \mathrm{~cm}^{-1}$. The quintet state appears to be too high-lying to detect by thermal population.

\section{Pseudopara-bis(phenylmethylenyl)[2.2]paracyclophane}

Pseudopara didiazo compound 5d was photolyzed similarly in an ESR cavity at $15 \mathrm{~K}$. Strong quintet signals due to dicarbẽne 5 were obtained: $|\mathrm{D}|=0.1213 \mathrm{~cm}^{-1}$ and $|\mathrm{E}|=0.0063 \mathrm{~cm}^{-1}$ (Fig. 9 ). In addition to these signals, multiplet signals due to a diradical species were observed at $\mathrm{ca}$. $330 \mathrm{mT}$ with the zero-field splitting parameters of $|\mathrm{D}|=0.0110 \mathrm{~cm}^{-1}$ and $|\mathrm{E}|$ $=0.00 \mathrm{~cm}^{-1}$. Additional signals at $464.4,530.1$ and $742.4 \mathrm{mT}$ are again attributed to a triplet monocarbene $\left(T_{G}\right)\left(|D|=0.3837 \mathrm{~cm}^{-1}\right.$ and $\left.|E|=0.0168 \mathrm{~cm}^{-1}\right)$. The intensity of the quintet signals decreased as the temperature was raised from 15 to $41 \mathrm{~K}$ obeying apparently Curie law. However, the intensity was not recovered when the temperature was lowered from $41 \mathrm{~K}$ back to $15 \mathrm{~K}$; about $60 \%$ of the original intensity was lost presumably by chemical reactions. The intensity of the diradical species increased during the annealing, showing that the quintet species may not be kinetically stable and converted to the diradical species even at these temperatures. Although it was not possible to obtain thermodynamical information from the temperature dependence of the signal intensities, the observation of the strong quintet signals strongly suggests that the quintet must be the ground state of dicarbene 5. A thermally populated triplet species was not detected in the temperature range $15 \sim 41 \mathrm{~K}$. The energy gap $\Delta E_{\mathrm{QT}}$ in $\underset{\sim}{5}$ appears to be even larger than that in $\underset{\sim}{3}$.
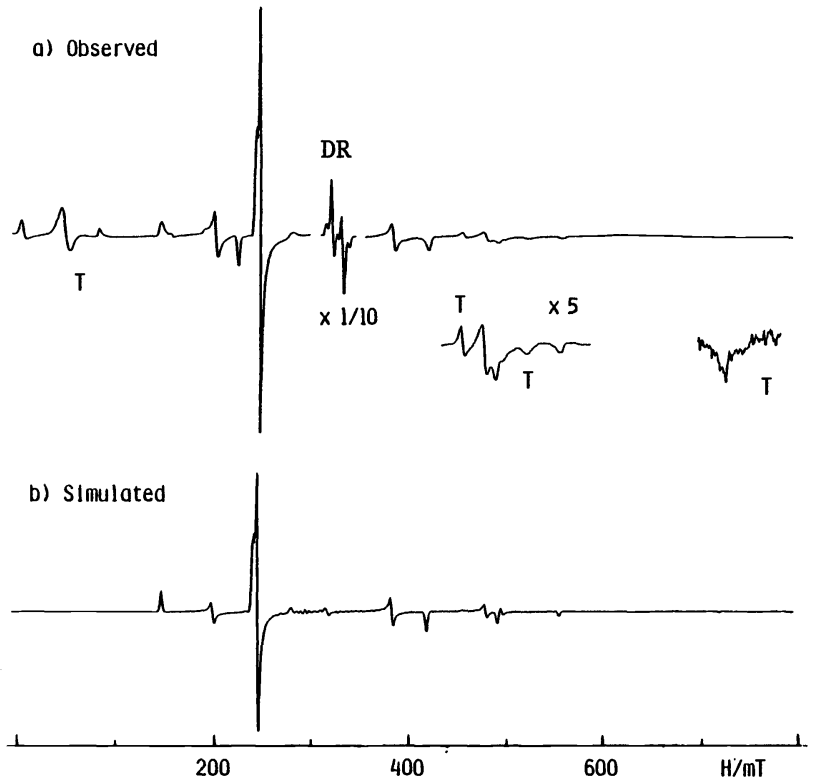

Fig. 9. ESR spectrum obtained by irradiation of pseudopara didiazo compound $5 \mathrm{~d}$ in $2-\mathrm{MTHF}$ at $11 \mathrm{~K}$.

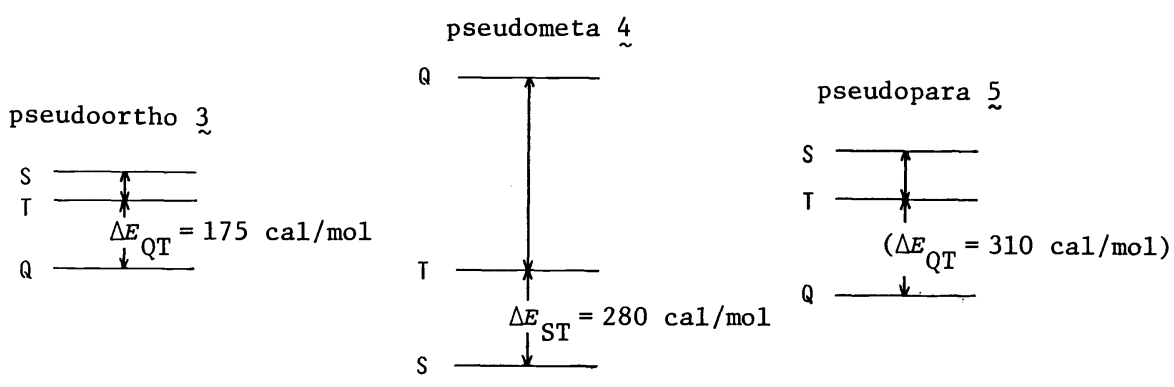

Fig. 10. The spin states of isomeric dicarbenes $\underset{\sim}{3} \sim \underset{\sim}{5}$. 
The difference in the spin states of isomeric [2.2] paracyclophane dicarbenes $3 \sim 5$ is summarized in Fig. 10. The results serve as the first operational test for the McC̃onñell's theory and lead to the conclusion that the mode of stacking of spin-containing benzene rings can be crucial in increasing the dimension of the intermolecular magnetic interaction of high-spin aromatic molecules in molecular assemblies.

\section{MAGNETIC INTERACTION AMONG DIPHENYLCARBENE MOLECULES GENERATED IN CRYSTALS OF CORRESPONDING DIAZODIPHENYLMETHANES}

The above findings have encouraged us to investigate intermolecular magnetic interaction among diphenylcarbene derivatives generated in the host crystals of the diazo precursors. favorable molecular interactions as the ortho and para stacking in Fig. 3 are realized in host crystals, intermoleuclar spin alignments will be established. We have scrutinized a series of substituted diazodiphenylmethanes and found two extreme examples (ref. 14).

\section{p-Methoxydiphenyldiazomethane, bis(p-methoxyphenyl)diazomethane and bis ( $p$ - octyloxyphenyl)diazomethane}

When microcrystalline samples of p-methoxydiphenyldiazomethane 6 a and bis(p-methoxyphenyl)diazomethane $7 \mathrm{a}$ were irradiated in an ESR cavity at $10 \mathrm{~K}$, the of $\tilde{s}$ erved spectra were entirely different from those of the triplet obtained in glassy matrices (Fig. 11), but resembled more closely that of the quintet dicarbene 5 (Fig. 9). Intense signals at 248.9 and $259.9 \mathrm{mT}$ for $6 \mathrm{a}$ and 247.6 and $259.0 \mathrm{mT}$ for $7 \mathrm{a}$ were observed immediately after the start of irradiation. These $\tilde{\tilde{C}}^{2}$ lines are characteristic of quintet species $(Q):|\mathrm{D}|=0.136 \pm 0.005$ and $|\mathrm{E}|=0.013 \pm$ $0.004 \mathrm{~cm}^{-1}$ for $6 \mathrm{a}$, and $|\mathrm{D}|=0.136 \pm 0.005$ and $|\mathrm{E}|=0.015 \pm 0.003 \mathrm{~cm}^{-1}$ for $7 \mathrm{a}$. The observation of the intense $\tilde{Q}^{\sim}$ signals suggests that pairs of triplet carbenes 6 and 7 generated in the crystals of $\underset{\sim}{6}$ a and $\underset{\sim}{7}$, respectively, interact efficiently to give the $Q \sim$ species. Weak

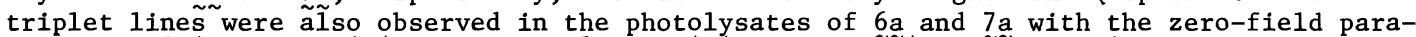
meters of $|\mathrm{D}|=0.408,|\mathrm{E}|=0.0180 \mathrm{~cm}^{-1}$, and $|\mathrm{D}|=0.408, \tau \tilde{\mathrm{E}} \mid=0 . \tilde{0} \tilde{1} 94 \mathrm{~cm}^{-1}$, respectively. These data are in good agreement with those obtained indisputably in 2-methyltetrahydrofuran glasses. Besides these lines, multiplet signals (M) were detected in the range $300 \sim 380 \mathrm{mT}$ (Fig. 11). The species responsible for $M$ is estimated to be molecular aggregates between the Q pairs. Thus the ESR spectral feature appears to be well-explained by postulating that various sizes of clusters of the triplet carbene species are formed by photolysis of the diazo compounds in crystals.

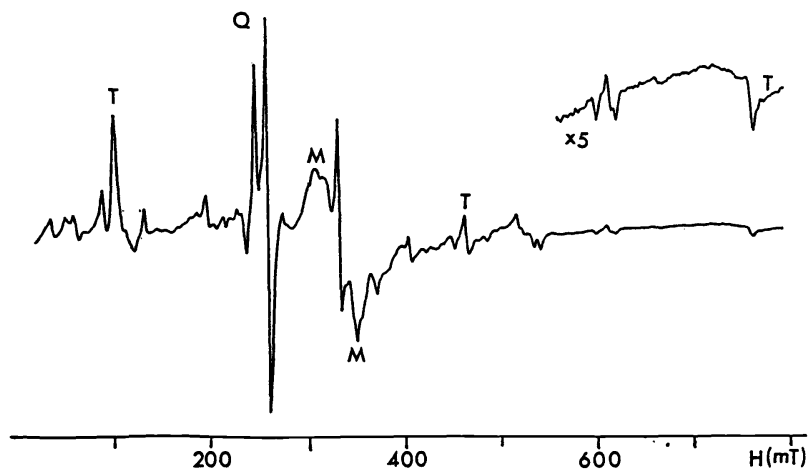

Fig. 11. ESR spectrum obtained by irradiation of the microcrystalline sample of bis (p-methoxyphenyl) diazomethane at $10 \mathrm{~K}$.

Temperature dependence of the intensities of the $M, Q$ and $T$ signals was studied in the range $14 \sim 120 \mathrm{~K}$. The Q species was kinetically stable up to $60 \mathrm{~K}$ and disappeared rapidly at $80 \mathrm{~K}$. The Curie plot of the $Q$ signals shows a concave departure from linearity, and is partly reproduced by a model in which $Q$ is the ground state and a thermally populated triplet lies about $30 \mathrm{cal} / \mathrm{mol}$ above the ground state. Most of the $\mathrm{T}$ signals observed is then reasoned to be due to the triplet carbene 7 isolated magnetically in the crystal.

Since the energy of the interaction between electron spins and an external magnetic field is smaller than the thermal energy, orientation of the spins, i.e., strength of magnetization, increases with that of the external field and decreases with temperature as dictated by the Brillouin function $B_{J}(x)$ :

$$
M=N g J \mu_{\mathrm{B}}^{B} \mathrm{~J}(\mathrm{x})
$$

where

$$
B_{J}(x)=\frac{2 J+1}{2 J} \operatorname{coth}\left(\frac{2 J+1}{2 J} x\right)-\frac{1}{2 J} \operatorname{coth}\left(\frac{x}{2 J}\right) \quad \text { and } x=g J \mu_{B} H /\left(k_{B} T\right)
$$




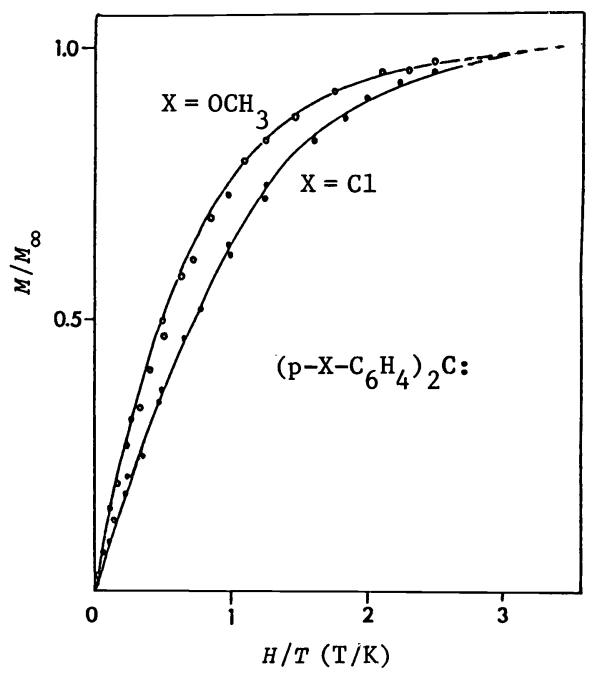

Fig. 12. Plots of magnetization of bis (pmethoxypheny 1 ) carbene (o) and bis ( $p$-chlorophenyl)carbene $(\bullet)$ generated in the corresponding diazo crystals vs temperaturenormalized field strength (ref. 14).

At very strong external magnetic field and very low temperature $(H / T \sim \infty)$, all the spins will be oriented to the direction of the magnetic field and saturation of magnetization will occur. When normalized to this value $M_{\infty}$, the magnetization curve should be equal to $B_{J}(x)$. Such plots for bis(p-methoxyphenyl)carbene $Z$ are shown in Fig. 12. Since 7 is composed of light atoms with small spin-orbit coupling, $J=S$ and $g=2$. The experimentai data are found by a non-linear least-square method to fit the theoretical curve with $S=(3.8 \pm 0.4) / 2$, providing independent evidence for the formation of a pair of triplet $?$ coupled ferromagnetically.

The ferromagnetic coupling of $\underset{\sim}{6}$ and $\underset{\sim}{7}$ generated in the precursor crystals of $\underset{\sim}{6 a}$ and $\underset{\sim}{7}$, respectively, is expected to arise from the intermolecular exchange interaction that $\tilde{\tilde{i}}$, effectively positive. The structure of the host crystals should be informative. Since it was difficult to obtain good single crystals amenable to an X-ray analysis, we studied the crystal structure $\left(\mathrm{P} 2{ }_{1} / a\right)$ of isomorphous $\mathrm{p}, \mathrm{p}^{\prime}$-dimethoxybenzophenone (ref. 16). Among four independent molecules in the unit cell, one benzene ring of the ketone molecule is nearly in parallel with the benzene ring of an adjacent molecule, taking a pseudopara-type orientation (see Fig. 3). Strictly speaking, the centers of the two interacting benzene rings are slight$1 y$ off and only two carbon atoms on one benzene ring are interacting effectively, but the stacking pattern satisfies McConnell's theory in a sense that the carbon atoms with positive spin density face those with negative ones at the interacting sites. The molecular packing in the crystal also rationalizes the presence of weak interaction between two carbene pairs each in the quintet state.

In order to design an assembly of carbene molecules that have intermolecular spin alignment over several molecules, we have taken advantage of the dispersion force of long alkyl chains (ref. 17). The ESR spectrum of the photolysate of polycrystalline bis(p-octyloxypheny1)diazomethane $8 \mathrm{\sim}$ at $10 \mathrm{~K}$ showed a complex multiplet in the range $260 \sim 380 \mathrm{mT}$, accompanied by

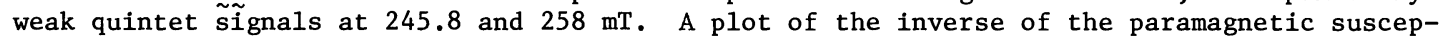
tibility vs temperature in the range $2 \sim 150 \mathrm{~K}$ consisted of four linear regions, showing development of various molecular fields at different temperatures (ref. 15). From the magnetization behavior at $2.1 \mathrm{~K}, S=8 / 2$ was obtained. Intermolecular magnetic interaction now develops over four carbene molecules.

\section{Bis(p-chlorophenyl)diazomethane}

When irradiated at $10 \mathrm{~K}$, monoclinic crystals of bis(p-chloropheny1)diazomethane showed strong triplet signals with the zero-field parameters $\left(|D|=0.3980\right.$ and $\left.|E|=0.0194 \mathrm{~cm}^{-1}\right)$ very close to those obtained in 2-MTHF glasses. A very weak signal assigned to a quintet was detected at $280 \mathrm{mT}$. From an analysis of the temperature dependence of these signals similar to those carried out for the methoxy derivatives, the carbene pair was found to be in the ground singlet state with the triplet and quintet states thermally populated. An X-ray analysis has revealed that the molecular packing is like the geminal overlap in Fig. 3 (ref. 14).

\section{CONCLUSION}

We have been able to demonstrate by model experiments that there are unique stacking patterns of the spin-distributed benzene rings of diphenylcarbene units leading to intermolecular electron spin alignment. They are ortho and para stacking as dictated by the McConnell's theory. When oriented by the dispersion force of long alkyl chains in the crystal of a diazo precursor, the spin alignment over four triplet molecules was achieved. 
Previously we showed that a large number of electron spins can be aligned systematically in organic molecules when properly designed as in polycarbenes $\frac{1}{\sim}$ and 2 . We are therefore in the position to take advantage of these guiding principles to develop $\tilde{h} i g h l y$ spin-aligned organic molecular systems that should have interesting magnetic properties.

\section{Acknowledgement}

It is a pleasure to acknowledge the full contributions of my coworkers at IMS, whose names occur in the references. I am also thankful to Prof. K. Itoh and his coworkers at Osaka City University for the collaboration and discussion throughout this work.

\section{REFERENCES}

1. W.T. Borden, Ed., Diradicals, Wiley, New York (1982).

2. A.R. Lepley and G.L.Closs, Eds., Chemically Induced Magnetic Polarization, Wiley, New York (1973).

3. Y. Teki, T, Takui, K. Itoh, H. Iwamura and K. Kobayashi, J. Am. Chem. Soc. 105, 37223723 (1983); Y. Teki, T. Takui, H. Yagi, K. Itoh and H. Iwamura, J. Chem. Phys. 83, 539547 (1985); Y. Teki, T. Takui, K. Itoh, H. Iwamura and K. Kobayashi, J. Am. Chem. Soc. 108, 2147-2156 (1986).

4. T. Sugawara, S. Bandow, K. Kimura, H. Iwamura and K. Itoh, J. Am. Chem. Soc. 106, 64496450 (1984); T. Sugawara, S. Bandow, K. Kimura, H. Iwamura and K. Itoh, J. Am. Chem. Soc. 108, 368-371 (1986).

5. H. Iwamura, Pure App1. Chem. 58, 187-196 (1986); H. Iwamura, Prodeedings of the 3rd International Kyoto Conference on New Aspects of Organic Chemistry, Kyoto, November; 1985; to be published from Elsevier.

6. T. Takui, K. Akashi, T. Kinoshita and K. Itoh, Abstracts of Papers, Third International Kyoto Conference on New Aspects of Organic Chemistry, Kyoto, November; 1985; p. 173.

7. A. Izuoka, S. Murata, T. Sugawara and H. Iwamura, J. Am. Chem. Soc. 107, 1786-1787 (1985),

8. H.M. McConnel1, J. Chem. Phys. 39, 1910 (1963).

9. K. Mukai, Bull. Chem. Soc. Jpn. 42, 42-46 (1969); K. Mukai and K. Ishizu, J. Phys. Soc. Jpn. 27, 783 (1969) ; K. Mukai, H. Nagai and K. Ishizu, Bull. Chem. Soc. Jpn. 48, 2381 -2382 (1975).

10. C.A. Hutchison, Jr. and B.E. Kohler, J. Chem. Phys. 51, 3327-3335 (1969); R.W. Brandon, G. L. Closs, C.E. Davoust, C.A. Hutchison,Jr., B.E. Kohler and R. Silbey, J. Chem. Phys. $\underline{43}$, 2006-2016 (1965).

11. K. Itoh, Pure App1. Chem. 50, 1251-1259 (1978).

12. H.J. Reich and D.J. Cram, J. Am. Chem. Soc. 91, 3527-3533 (1969).

13. K. Itoh, Chem. Phys. Lett. $\frac{1}{1}, 235-238$ (1967); Y. Teki, T. Takui, K. Itoh, A. Izuoka, S, Murata, T. Sugawara and $\mathrm{H}$. Iwamura, to be published.

14. T. Sugawara, H. Tukada, A. Izuoka, S. Murata and H. Iwamura, J. Am. Chem. Soc. in press.

15. T. Sugawara, S. Murata, K. Kimura, H. Iwamura, Y. Sugawara and H. Iwasaki, J. Am. Chem. Soc. 107, 5293-5294 (1985).

16. I.L. Karle, H. Hauptman, L. Karle and A.B. Wing, Acta Crystallogr. 10, 481-483 (1957).

17. See for example, S. Abrahamsson, Acta Crystallogr. 12, 301-304 (1959). 